\title{
Two-Dimensional Fuzzy Sliding Mode Control of a Field-Sensed Magnetic Suspension System
}

\author{
Jen-Hsing $\mathrm{Li}^{1}$ and Juing-Shian Chiou ${ }^{2}$ \\ ${ }^{1}$ Department of Electrical Engineering, Kun Shan University, Tainan City, Taiwan \\ ${ }^{2}$ Department of Electrical Engineering, Southern Taiwan University of Science and Technology, Tainan City 710, Taiwan \\ Correspondence should be addressed to Juing-Shian Chiou; jschiou@mail.stust.edu.tw
}

Received 18 February 2014; Accepted 15 April 2014; Published 12 May 2014

Academic Editor: Her-Terng Yau

Copyright (c) 2014 J.-H. Li and J.-S. Chiou. This is an open access article distributed under the Creative Commons Attribution License, which permits unrestricted use, distribution, and reproduction in any medium, provided the original work is properly cited.

\begin{abstract}
This paper presents the two-dimensional fuzzy sliding mode control of a field-sensed magnetic suspension system. The fuzzy rules include both the sliding manifold and its derivative. The fuzzy sliding mode control has advantages of the sliding mode control and the fuzzy control rules are minimized. Magnetic suspension systems are nonlinear and inherently unstable systems. The twodimensional fuzzy sliding mode control can stabilize the nonlinear systems globally and attenuate chatter effectively. It is adequate to be applied to magnetic suspension systems. New design circuits of magnetic suspension systems are proposed in this paper. ARM Cortex-M3 microcontroller is utilized as a digital controller. The implemented driver, sensor, and control circuits are simpler, more inexpensive, and effective. This apparatus is satisfactory for engineering education. In the hands-on experiments, the proposed control scheme markedly improves performances of the field-sensed magnetic suspension system.
\end{abstract}

\section{Introduction}

Sliding mode control (SMC) is a powerful nonlinear control technique $[1,2]$. SMC applies a discontinuous control signal that forces the system trajectory to slide along the boundaries of the control structures. The controlled system is switched from one continuous structure to another. Hence, sliding mode control is a variable structure control methodology. SMC has low sensitivity to the plant parameter uncertainties and disturbances. SMC can apply to a class of nonlinear systems in the global sense and consider robustness issues for modeling uncertainties and disturbances. The disadvantage of SMC is the problem of chattering.

Recently, applications of the fuzzy sliding mode control (FSMC) are popular $[3,4]$. The FSMC can reduce the chattering problem. The FSMC integrates the sliding mode control (SMC) and the fuzzy logic control (FLC). The fuzzy set theory was introduced by Wang [5]. The FLC methods have been investigated for almost 50 years. They have been successfully adopted for many engineering applications. The FSMC provides a minimal fuzzy set of the FLC and a systematic design procedure $[3,4]$. It provides a simple way to achieve asymptotic stability of the system. In general, FSMC fuzzifies the sliding manifold to improve the chattering problem caused by the switching in the control input. This is the same idea applied in the present work. But the fuzzy control part of FSMC is reinforced by the two-dimensional fuzzy control in this paper. The advantages are more flexible to design the fuzzy control part and more effective to reduce the system chattering. The two-dimensional fuzzy sliding mode control (2DFSMC) improves the problem of chattering effectively. The disadvantage of 2DFSMC is the computational load.

Magnetic suspension techniques have been widely used in different fields of industrial applications such as levitation of high speed trains [6,7], frictionless bearings [8], and magnetically suspended wind tunnels [9]. Magnetic suspension systems (MSS) are nonlinear and inherently unstable. Hence, they are good apparatus for verifying different control techniques. Magnetic suspension systems are appropriate educational tools in engineering courses [1024]. Cho et al. [10] provided an experimental comparison between a sliding mode controller and a classical controller for a magnetic levitation system (MLS). As expected, 
the sliding mode controller provided perfect performance. Shen [11] compared the $H_{\infty}$ control, the sliding mode control, and the PID control of the MLS. From experimental results of Shen [11], these three controllers were excellent at specified conditions. Al-Muthairi and Zribi [12] addressed specified dynamic sliding mode control of a MLS. From simulation results, the proposed control scheme reduced the chattering problem. Yang et al. [13] proposed the dynamic surface control a voltage-controlled MLS. The developed dynamic surface control is modified and applied to the system under study. Kuo et al. [14] presented the design of a novel fuzzy sliding-mode control (NFSMC) for the magnetic ball levitation system. A NFSMC scheme was an integration of the SMC and the FSMC with an adjustable gain. Simulation results suggested that the proposed scheme is feasible for the magnetic ball levitation system. Chiang et al. [15] proposed an integral variable-structure grey control for a MLS. The proposed controller does not require exact knowledge of the maximum uncertainly boundary for disturbances and parameter variations. Experimental results therein showed good performance and reduces the chattering phenomenon. Chen et al. [16] proposed the fuzzy sliding mode control of a magnetic ball suspension system. The adaptive fuzzy estimator was designed to estimate the unknown lumped uncertainty. It reduced the chattering magnitude or steady state error phenomenon. Lin et al. [17, 18] proposed two SMC and neural network controllers of the MLS. In [17], the sliding-mode control system using a radial basis function network (RBFN) is proposed to control the position of a levitated object of a MLS. In [18], a robust dynamic sliding mode control system using a recurrent Elman neural network (RENN) is proposed to control the position of a levitated object of a MLS. The RBFN is utilized to estimate the uncertain system dynamics online. The RENN is used to estimate the unknown nonlinear function of the MLS. Both stabilities of these controls were guaranteed by way of Lyapunov stability analysis. The experimental results were included in these papers. Li [19] proposed a fuzzy supervisory control of a DSP-based MLS. The discrete-time model of the MLS is derived and the stability is guaranteed by the root locus methodology. A fuzzy supervisory control is used to compensate for system nonlinearities. $\mathrm{Li}$ and $\mathrm{Wu}$ [20] proposed the two-dimensional fuzzy sliding mode control (2DFSMC) of the MLS. Now, the same idea is applied to the field-sensed magnetic suspension system (FSMSS) in this paper. The more precise derivations are proposed. With respect to the FSMSS, novel circuits are proposed. The implemented driver, sensor, and control circuits are more simple, inexpensive, and effective. Shieh et al. [21] proposed a robust optimal sliding-mode control approach for position tracking of a MLS. The feedback linearization method is used and the integral SMC and $H_{\infty}$ control were based on linearized model. The experimental results verified the proposed control scheme.

Lundberg et al. [22] used inexpensive experiments to teach the analysis and design of magnetic levitation in undergraduate control courses. The analog control of the FSMSS is a class project at MIT (Massachusetts Institute of Technology) on feedback systems. Subsequently, Li [23] proposed multiloop PID control for the FSMSS. Two control loops are implemented. The control scheme is mathematically analyzed by the Routh stability criterion. The stability of the FSMSS is guaranteed and the experimental result is accomplished successfully. Later, the FSMSS is extended to a discrete-time control scheme [24], and the problem of robust stability on discrete-time systems is addressed. The size of allowable perturbation in polynomial coefficient space was estimated for the output feedback control of the FSMSS. This paper presents the 2DFSMC of the FSMSS. The sliding manifold and its derivative are both fuzzied for fuzzy logic rules. The more precise derivations are proposed. For the hands-on experiments, this proposed control scheme improves performances of the FSMSS.

This paper is organized as follows. The SMC control methodology is presented in Section 2. The FSMC control technique is stated in Section 3. The presented 2DFSMC control strategy is delineated in Section 4. The hands-on experiments are discussed in Section 5. The conclusion is provided in Section 6.

\section{Sliding Mode Control}

The control model of a FSMSS is shown in the following [19, $20,23,24]$ :

$$
m \frac{d^{2} x}{d t^{2}}=m g-\frac{i^{2}}{f(x)}
$$

where $m$ is the mass of the magnet, $x$ is the distance between the electromagnet and the magnet, $i$ is the coil current, and $g$ is the gravitational acceleration. The electromagnetic force is the function of $i^{2} / f(x)$. Let the distance $(x)$ be the function of the measurement output $(v)$ of the magnet position $x(v)$. If the sensor circuit is linear, for example $x(v)=c \cdot v, c$ is a constant. Then $d x / d v=c$ and $d^{2} x / d v^{2}=0$ and (1) is rewritten as follows:

$$
\widehat{m} \frac{d^{2} v}{d t^{2}}=m g-\frac{i^{2}}{\widehat{f}(v)}
$$

where $\hat{f}(v)=f(x(v))$ is the approximate polynomial function and $\widehat{m}=m(d x / d v)$ (if $x(v)=c \cdot v$ then $\widehat{m}=m \cdot c$ ). Let $v_{1}=v$ be the position measurement, $v_{2}=\dot{v}$ its derivative, and control input $u=i$ the coil current. The nonlinear state equation of a FSMSS is

$$
\begin{aligned}
& \dot{v}_{1}=v_{2}, \\
& \dot{v}_{2}=\frac{m}{\widehat{m}} g-\frac{u^{2}}{\widehat{m} \widehat{f}\left(v_{1}\right)} .
\end{aligned}
$$

The control objective is to control the levitation height $v(t)$ at the desired height $v_{0}(t)$. Let the tracking error be

$$
\varepsilon(t)=v_{1}(t)-v_{0}(t)
$$

with respect to the tracking control, an integral error term is included in the sliding manifold as follows:

$$
\xi(t)=\dot{\varepsilon}(t)+\lambda_{1} \varepsilon(t)+\lambda_{2} \int_{0}^{t} \varepsilon(\tau) d \tau .
$$


For the SMC, the approaching and sliding conditions of the sliding manifold $\xi(t)=0$ is

$$
\xi(t) \dot{\xi}(t)<0 .
$$

By Lyapunov stability criterion, if the above condition holds, the asymptotical stability is guaranteed for nonlinear systems. The derivative of $\xi(t)$ is given as follows:

$$
\begin{aligned}
\dot{\xi}(t) & =\ddot{\varepsilon}+\lambda_{1} \dot{\varepsilon}+\lambda_{2} \varepsilon \\
& =\left(\dot{v}_{2}-\ddot{v}_{0}\right)+\lambda_{1}\left(v_{2}-\dot{v}_{0}\right)+\lambda_{2}\left(v_{1}-v_{0}\right) \\
& =\frac{m}{\widehat{m}} g-\frac{u^{2}}{\widehat{m} \hat{f}\left(v_{1}\right)}-\ddot{v}_{0}+\lambda_{1}\left(v_{2}-\dot{v}_{0}\right)+\lambda_{2}\left(v_{1}-v_{0}\right) .
\end{aligned}
$$

The equivalent control law for $\dot{\xi}(t)=0$ can be obtained as follows $[1,2,10]$ :

$$
u_{\mathrm{eq}}^{2}=\widehat{m} \widehat{f}\left(v_{1}\right)\left\{\frac{m}{\widehat{m}} g-\ddot{v}_{0}+\lambda_{1}\left(v_{2}-\dot{v}_{0}\right)+\lambda_{2}\left(v_{1}-v_{0}\right)\right\}
$$

and the complete sliding mode control $u_{\mathrm{sm} 1}$ is given as follows:

$$
\begin{aligned}
u_{\mathrm{sm} 1}^{2}=\widehat{m} \hat{f}\left(v_{1}\right)\{ & \frac{m}{\widehat{m}} g-\ddot{v}_{0}+\lambda_{1}\left(v_{2}-\dot{v}_{0}\right) \\
& \left.+\lambda_{2}\left(v_{1}-v_{0}\right)+\eta_{1} \cdot \operatorname{sign}(\xi(t))\right\},
\end{aligned}
$$

where $\eta_{1}$ is a positive real number and the sign function $\operatorname{sign}(\cdot)$ is

$$
\operatorname{sign}(\xi(t))= \begin{cases}1, & \text { if } \xi(t)>0 \\ -1, & \text { if } \xi(t)<0 \\ 0, & \text { if } \xi(t)=0 .\end{cases}
$$

Substituting (9) into $\xi(t) \dot{\xi}(t)$, we can obtain the following inequality

$$
\xi(t) \dot{\xi}(t)=-\eta_{1}|\xi(t)|<0 .
$$

Therefore the controller (9) satisfies the approaching and sliding conditions (6). The error $\varepsilon(t)$ and the error integral $\int_{0}^{t} \varepsilon(\tau) d \tau$ approach to zero for steady states. One possible method of improving the chattering problem is to replace the sign function $\operatorname{sign}(\cdot)$ with the saturation function sat $(\cdot)$ as follows $[1,2,10]$ :

$$
\begin{aligned}
u_{\mathrm{sm} 2}^{2}=\widehat{m} \widehat{f}\left(v_{1}\right)\left\{\frac{m}{\widehat{m}}\right. & g-\ddot{v}_{0}+\lambda_{1}\left(v_{2}-\dot{v}_{0}\right) \\
& \left.+\lambda_{2}\left(v_{1}-v_{0}\right)+\eta_{2} \cdot \operatorname{sat}\left(\frac{\xi(t)}{\phi}\right)\right\},
\end{aligned}
$$

where

$$
\operatorname{sat}\left(\frac{\xi(t)}{\phi}\right)= \begin{cases}\operatorname{sign}(\xi(t)), & \text { if }|\xi(t)| \geq \phi \\ \frac{\xi(t)}{\phi}, & \text { if }|\xi(t)|<\phi,\end{cases}
$$

$\eta_{2}$ is a positive real number and $\phi$ is the band parameter and is a positive real number.
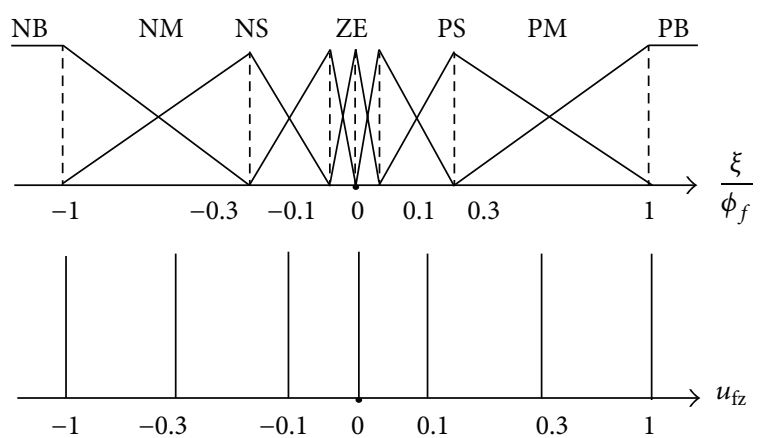

FIGURE 1: The membership functions of the sliding manifold and the fuzzy control.

\section{Fuzzy Sliding Mode Control}

In this section, the general FSMC is reviewed. From (9), the FSMC is determined by

$$
\begin{aligned}
u_{\mathrm{fsm}}^{2}=\widehat{m} \widehat{f}\left(v_{1}\right)\left\{\frac{m}{\widehat{m}} g-\ddot{v}_{0}+\lambda_{1}\left(v_{2}-\dot{v}_{0}\right)\right. \\
\left.+\lambda_{2}\left(v_{1}-v_{0}\right)+\eta_{f} \cdot u_{\mathrm{fz}}\left(\frac{\xi}{\phi_{f}}\right)\right\},
\end{aligned}
$$

where $\eta_{f}$ is the denormalized factor of FSMC and $\phi_{f}$ is the normalized factor of $\xi$. The fuzzy inference rules are generally determined as follows:

Rule 1: IF $\xi / \phi_{f}$ is $\mathrm{PB}$, THEN $u_{\mathrm{fz}}$ is $\mathrm{PB}$,

Rule 2: IF $\xi / \phi_{f}$ is PM, THEN $u_{\mathrm{fz}}$ is $\mathrm{PM}$,

Rule 3: IF $\xi / \phi_{f}$ is PS, THEN $u_{\mathrm{fz}}$ is PS,

Rule 4: IF $\xi / \phi_{f}$ is ZE, THEN $u_{\mathrm{fz}}$ is $\mathrm{ZE}$,

Rule 5: IF $\xi / \phi_{f}$ is NS, THEN $u_{\mathrm{fz}}$ is NS,

Rule 6: IF $\xi / \phi_{f}$ is NM, THEN $u_{\mathrm{fz}}$ is NM,

Rule 7: IF $\xi / \phi_{f}$ is NB, THEN $u_{\mathrm{fz}}$ is NB.

The linguistic sets of the universe of discourse $\xi / \phi_{f}$ and the fuzzy control output $u_{\mathrm{fz}}$ have the forms, positive big $(\mathrm{PB})$, positive middle $(\mathrm{PM})$, positive small $(\mathrm{PS})$, zero $(\mathrm{ZE})$, negative small (NS), negative middle (NM), and negative big (NB). The membership functions are shown in Figure 1. The centroid defuzzification method can be expressed by the following equation:

$$
u_{\mathrm{fz}}=\frac{\sum_{n=1}^{7} \mu_{n}\left(\xi / \phi_{f}\right) \times u_{\mathrm{fz}}(n)}{\sum_{n=1}^{7} \mu_{n}\left(\xi / \phi_{f}\right)}
$$


where $n$ is the rule number and $\mu_{n}(\cdot)$ is the membership function. Substituting (14) into $\xi(t) \dot{\xi}(t)$, we can obtain the following inequality:

$$
\begin{aligned}
& \xi(t) \dot{\xi}(t) \\
&=\xi(t) \cdot\left\{\frac{m}{\widehat{m}} g-\frac{u_{\mathrm{fsm}}^{2}}{\widehat{m} \widehat{f}\left(v_{1}\right)}-\ddot{v}_{0}+\lambda_{1}\left(v_{2}-\dot{v}_{0}\right)\right. \\
&\left.+\lambda_{2}\left(v_{1}-v_{0}\right)\right\} \\
&=-\eta_{f} \cdot \xi \cdot u_{\mathrm{fz}}\left(\frac{\xi}{\phi_{f}}\right) \leq 0 .
\end{aligned}
$$

From Rule 1 to Rule 7, the sliding manifold $\xi$ and fuzzy control $u_{\mathrm{fz}}$ are in phase. Hence, the controller (14) satisfies the approaching and sliding conditions (6).

\section{Two-Dimensional Fuzzy Sliding Mode Control}

In this section, the two-dimensional fuzzy sliding mode control (2DFSMC) is reviewed and presented. The 2DFSMC technique is to use both the sliding manifold $\xi$ and its derivative $\dot{\xi}$, simultaneously. Similarly from (9), (12), and (14), the $2 \mathrm{DFSMC}$ is given as follows:

$$
\begin{gathered}
u_{2 \mathrm{fsm}}^{2}=\widehat{m} \widehat{f}\left(v_{1}\right)\left\{\frac{m}{\widehat{m}} g-\ddot{v}_{0}+\lambda_{1}\left(v_{2}-\dot{v}_{0}\right)+\lambda_{2}\left(v_{1}-v_{0}\right)\right. \\
\left.+\eta_{2 f} \cdot u_{2 \mathrm{fz}}\left(\frac{\xi}{\phi_{f_{1}}}, \frac{\dot{\xi}}{\phi_{f_{2}}}\right)\right\},
\end{gathered}
$$

where $u_{2 \mathrm{fz}}\left(\xi / \phi_{f_{1}}, \dot{\xi} / \phi_{f_{2}}\right)$ is the fuzzy function of $\xi / \phi_{f_{1}}$ and $\dot{\xi} / \phi_{f_{2}} \cdot \eta_{2 f}$ is the denormalized factor, $\phi_{f_{1}}$ is the normalized factor of $\xi$, and $\phi_{f_{2}}$ is the normalized factor of $\dot{\xi}$. The membership functions of fuzzy numbers $\xi / \phi_{f_{1}}, \dot{\xi} / \phi_{f_{2}}$, and $u_{2 \text { fz }}$ and the linguistic states PB, PM, PS, ZE, NS, NM, and NB are shown in Figure 2. The design rules are to make the derivative of Lyapunov function negative. They can be represented in Table 1 . In the previous section, only one proposition $\xi / \phi_{f}$ is concerned. It is named one-dimensional fuzzy sliding mode control. Taking Table 1 into account, both of $\xi / \phi_{f_{1}}$ and $\dot{\xi} / \phi_{f_{2}}$ are discussed. Therefore, it is named 2DFSMC. For the defuzzification, the same centroid defuzzification method is utilized as follows:

$$
u_{2 \mathrm{fz}}=\frac{\sum_{n=1}^{49} \mu_{n}\left(\left(\xi / \phi_{f_{1}}\right) \times\left(\dot{\xi} / \phi_{f_{2}}\right)\right) \times u_{2 \mathrm{fz}}(n)}{\sum_{n=1}^{49} \mu_{n}\left(\left(\xi / \phi_{f_{1}}\right) \times\left(\dot{\xi} / \phi_{f_{2}}\right)\right)}
$$
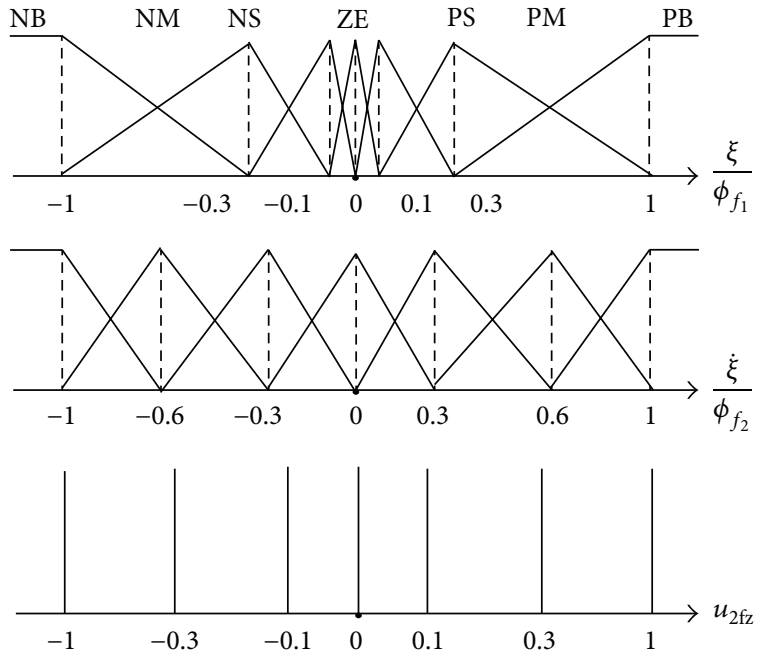

FIgURE 2: The membership functions of the 2DFSMC.

where the inferred membership function is $\mu_{n}\left(\left(\xi / \phi_{f_{1}}\right) \times\right.$ $\left.\left(\dot{\xi} / \phi_{f_{2}}\right)\right)=\min \left\{\mu_{n}\left(\xi / \phi_{f_{1}}\right), \mu_{n}\left(\dot{\xi} / \phi_{f_{2}}\right)\right\}$. The total number of possible nonconflicting fuzzy inference rules is 49 . Therefore, the fuzzy conclusion of $u_{2 \mathrm{fz}}$ has the following property:

$$
\begin{aligned}
& u_{2 \mathrm{fz}}>0, \quad \text { if } \frac{\xi}{\phi_{f_{1}}}+\frac{\dot{\xi}}{\phi_{f_{2}}}>0, \\
& u_{2 \mathrm{fz}}=0, \quad \text { if } \frac{\xi}{\phi_{f_{1}}}+\frac{\dot{\xi}}{\phi_{f_{2}}}=0, \\
& u_{2 \mathrm{fz}}<0, \quad \text { if } \frac{\xi}{\phi_{f_{1}}}+\frac{\dot{\xi}}{\phi_{f_{2}}}<0 .
\end{aligned}
$$

Substituting (17) into $\xi(t) \dot{\xi}(t)$, the derivative of the Lyapunov function $(1 / 2) \xi^{2}(t)$ can be obtained:

$$
\begin{aligned}
& \xi(t) \dot{\xi}(t)=\xi(t) \cdot\left\{\frac{m}{\widehat{m}} g-\frac{u_{2 \mathrm{fsm}}^{2}}{\widehat{m} \widehat{f}\left(v_{1}\right)}-\ddot{v}_{0}\right. \\
& \left.+\lambda_{1}\left(v_{2}-\dot{v}_{0}\right)+\lambda_{2}\left(v_{1}-v_{0}\right)\right\} \\
& =-\eta_{2 f} \cdot \xi \cdot u_{2 \mathrm{fz}}\left(\frac{\xi}{\phi_{f_{1}}}, \frac{\dot{\xi}}{\phi_{f_{2}}}\right) .
\end{aligned}
$$

Now, we focus on the rule table. Let the rule base Table 1 be viewed as a phase plane of fuzzy numbers; the horizontal axis is $\xi$ and the vertical axis is the derivative of $\xi$. The diagonal linguistic states are all ZE; then the diagonal line is

$$
\frac{\xi}{\phi_{f_{1}}}+\frac{\dot{\xi}}{\phi_{f_{2}}}=0 .
$$

From the rule base Table 1, the upper triangular linguistic states are all positive and the lower triangular linguistic states are all negative. By rearranging (21), we can obtain

$$
\dot{\xi}(t)=-\frac{\phi_{f_{2}}}{\phi_{f_{1}}} \xi(t) .
$$


TABLE 1: Rules of the 2DFSMC.

\begin{tabular}{cccccccc}
\hline$u_{2 f z}$ & & & $\xi / \phi_{f 1}$ & & & \\
& NB & NM & NS & ZE & PS & PM & PB \\
\hline$\dot{\xi} / \phi_{f_{2}}$ & & & & & PB & PB & PB \\
PB & ZE & PS & PM & PM & PM & PM & PB \\
PM & NS & ZE & PS & PS & PS & PM & PB \\
PS & NM & NS & ZE & PS & PS & PS & PM \\
ZE & NM & NS & NS & ZE & ZE & PS & PM \\
NS & NB & NM & NS & NS & NS & ZE & PS \\
NM & NB & NM & NM & NS & NM & NS & ZE \\
NB & NB & NB & NB & NM &
\end{tabular}

Substituting (22) into $\xi(t) \dot{\xi}(t)$, we can obtain the derivative of the Lyapunov function $(1 / 2) \xi^{2}(t)$ as follows:

$$
\xi(t) \cdot \dot{\xi}(t)=-\frac{\phi_{f_{2}}}{\phi_{f_{1}}} \xi^{2}(t) \leq 0 .
$$

Hence, the 2DFSMC controller (17) satisfies the approaching and sliding conditions (6). The asymptotical stability is guaranteed and the system chattering is reduced.

\section{Hands-On Experiments}

In this section, the hands-on experiments will be presented. The setup of the FSMSS experiment is shown in Figure 3. The driver and sensor circuits are shown in Figure 4. These circuits are new designed for the FSMSS. The circuit of Figure 4(a) is the coil current sensor and driver. The MOSFET IRF1010E is served as a switch of pulse width modulation (PWM). The IR2101S is a MOSFET trigger chip. This switch element is placed on the top of the driver circuit. The current sensing resistor is placed on the bottom of the driver circuit. We can use an inexpensive element in this circuit. Otherwise we must use an expensive current sensor. The PWM control is from the Arminno [25] PWM port and the frequency of PWM is $17.6 \mathrm{KHz}$. Arminno is a 32-bit microcontroller board based on ARM Cortex-M3 processor. The ARM Cortex-M3 processor is the industry-leading 32-bit processor nowadays. It is suitable to develop high-performance lowcost platforms for industrial control systems. The $\mu$ Vision 4 development environment [26] helps us quickly create and test embedded applications for the Cortex-M processor. The $\mu$ Vision 4 includes a text-editor, a C-complier, an assembler, a debugger, and a download tool. Users can realize control theories by use of C-programs. In our hands-on experiments, the programs are designed by C-language and based on $\mu$ Vision4 development environment.

When the MOSFET is off, the flywheel diode (1N5819) can maintain coil current circuit. It can avoid the open-circuit spark. The $0.2 \Omega$ resistor transfers current signal to voltage signal. The coil current signal is sampled by the Arminno analog to digital (A/D) port. The sample rate is $1 \mathrm{KHz}$. The circuit of Figure 4(b) is the magnet position sensor circuit. The A1321 is a magnetic field sensor which has a ratiometric output voltage. It varies in proportion to the strength of
TABLE 2: Measured data of input/output signals.

\begin{tabular}{lcc}
\hline Set point & Position $(\mathrm{V})$ & Current $(\mathrm{V})$ \\
\hline Number 1 & 0.5 & 0.66 \\
Number 2 & 1.0 & 1.16 \\
Number 3 & 1.5 & 1.5 \\
Number 4 & 2.0 & 1.68 \\
Number 5 & 2.5 & 1.83 \\
\hline
\end{tabular}

the magnetic field. The noninverting amplifier is adjusted so that the magnet position signal varies from 0 Volts to 3 Volts. When the magnet is placed on the top of A1321, the output voltage can be measured as 3 Volts. The output voltage is decreasing while the magnet is pulled away from the A1321. So we can measure the position of the magnet by this circuit. From Figure 5, the bottom of the magnet is exactly $1 \mathrm{~cm}$ away from the magnetic field sensor; then the measured voltage is 1.5 Volts and the distance between the electromagnet and the top of the magnet is $4 \mathrm{~cm}\left(x_{0}=4 \mathrm{~cm}\right)$. The bias current required to balance the gravitational force is $0.5 \mathrm{Amps}$.

The block diagram of a FSMSS is shown in Figure 6. Two control loops are utilized in Figure 6. The coil current is compensated in the inner loop and the magnet position is stabilized in the outer loop. The coil current is well designed by digital PI (proportional and integral) controller $[19,20$, 24]. Figure 7 is from the digital storage oscilloscope. This picture shows the step response of the coil current signal from 1 Volt to 2 Volts. The vertical scale is $500 \mathrm{mV} / \mathrm{Div}$ and the horizontal scale is $10 \mathrm{~ms} / \mathrm{Div}$. From Figure 7, the settling time of the coil current is about $25 \mathrm{~ms}$. The dynamics of coil current are faster than that of position, so the inner loop subsystem can be approximated as a constant gain current driver. Then the inner loop design is finished.

Now, the second hands-on experiment is to measure the characteristic curve of the FSMSS. From $[19,20,24]$, the digital PD (proportional and derivative) control can be used to stabilize the FSMSS. We choose five equilibrium set points and find the corresponding stable bias coil current against gravity. By experiments, the corresponding stable input-output data are shown in Table 2. By Curve Fitting Tool of MATLAB, the characteristic curve is shown in Figure 8. The $x$-axis is the position measurement and the $y$-axis is 


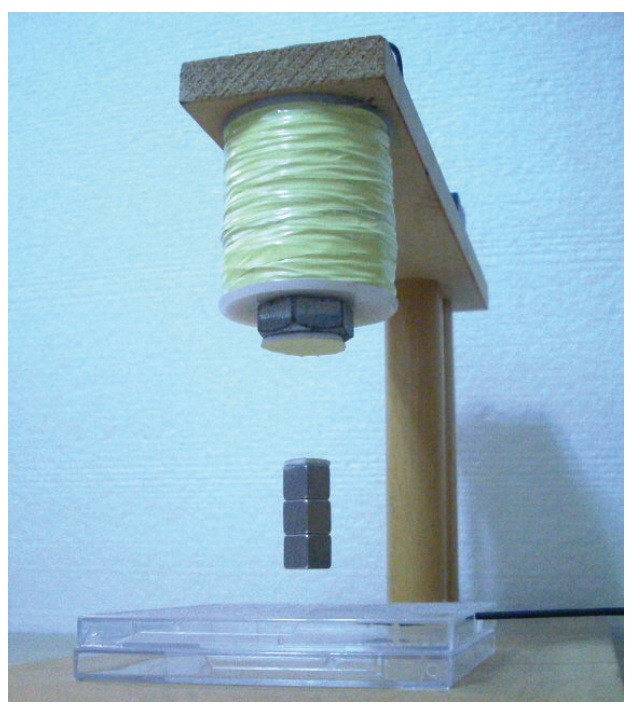

FIgURE 3: A photograph of a FSMSS.

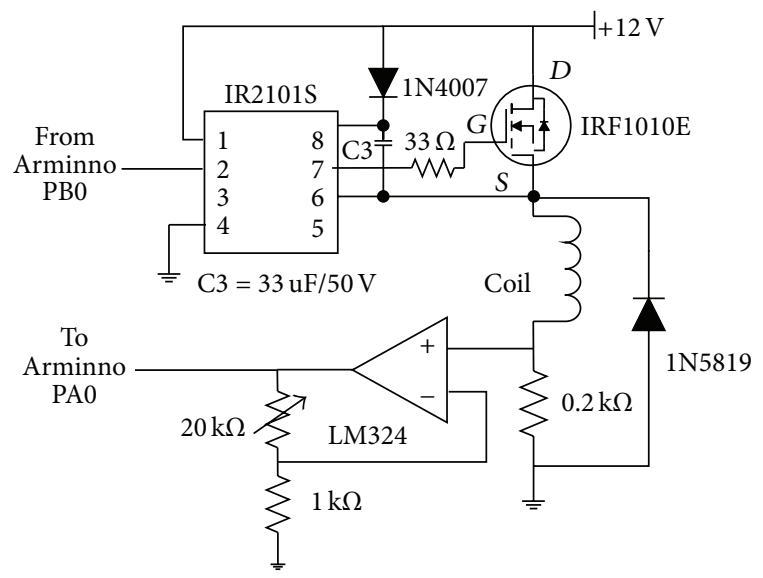

(a)

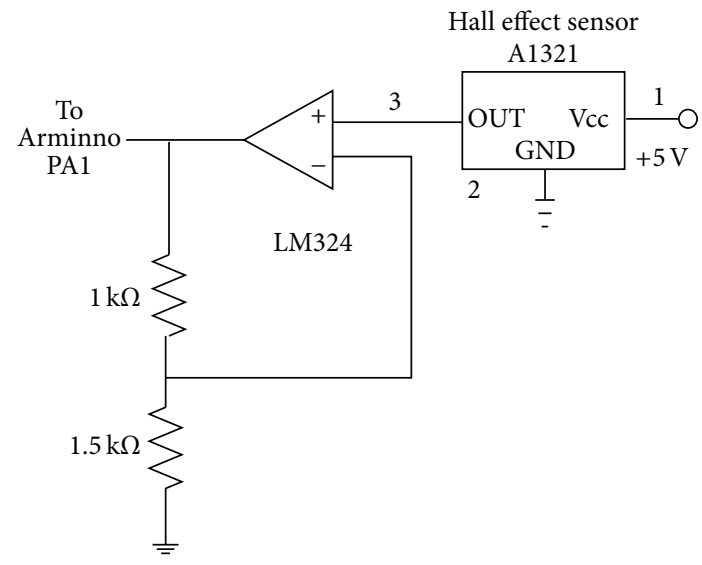

(b)

FIgURE 4: The implemented circuits of a FSMSS.

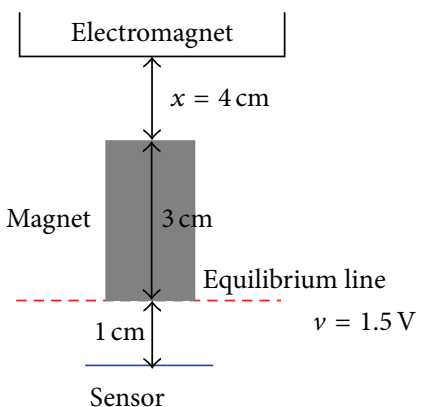

FIgURE 5: The motion direction of a FSMSS.

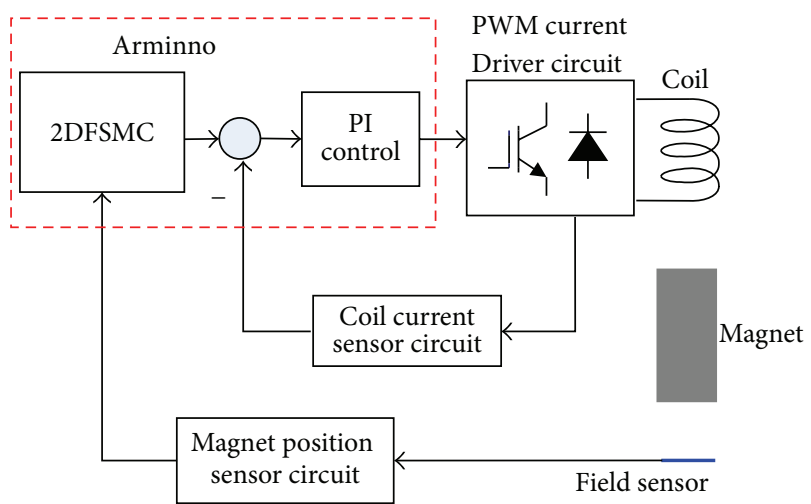

FIGURE 6: 2DFSMC of a FSMSS.

the square of coil current. The fitting curve of Figure 8 is the 4th degree of polynomial and is presented as follows:

$$
i^{2}=m g \hat{f}(v)=p_{1} v^{4}+p_{2} v^{3}+p_{3} v^{2}+p_{4} v+p_{5},
$$

where the coefficients of the polynomial are $p_{1}=0.4083, p_{2}=$ $-2.477, p_{3}=4.867, p_{4}=-1.912$, and $p_{5}=0.4589$.

The third hands-on experiment presents the 2DFSMC. In our experiments, the velocity of the magnet is not available. Hence, the approximation $v_{2} \approx \Delta v_{1}(k) / \Delta t=\left(v_{1}(k)-v_{1}(k-\right.$ $1)) / \Delta t$ is made, where $\Delta t$ is the sampling period. Therefore (5) and (12) are modified as follows:

$$
\widetilde{\xi}(k)=\frac{\Delta \varepsilon(k)}{\Delta t}+\lambda_{1} \varepsilon(k)+\lambda_{2} \sum_{n=0}^{k} \varepsilon(n) \cdot \Delta t,
$$

where $\Delta \varepsilon(k)=\varepsilon(k)-\varepsilon(k-1), \varepsilon(k)$ is the present error, and $\varepsilon(k-1)$ is the previous error. Consider

$$
\begin{aligned}
u_{\mathrm{sm} 2}^{2}(k)=m g \widehat{f}\left(v_{1}\right)\{1 & +\frac{\lambda_{1}}{g} \frac{\widehat{m}}{m} \frac{\Delta v_{1}(k)}{\Delta t}+\frac{\lambda_{2}}{g} \frac{\widehat{m}}{m} \varepsilon(k) \\
& \left.+\frac{\eta_{2}}{g} \frac{\widehat{m}}{m} \operatorname{sat}\left(\frac{\tilde{\xi}(k)}{\phi}\right)\right\} \\
= & m g \widehat{f}\left(v_{1}\right)\left\{1+\lambda_{1}^{\prime} \Delta v_{1}(k)+\lambda_{2}^{\prime} \varepsilon(k)\right. \\
& \left.+\eta_{2}^{\prime} \operatorname{sat}\left(\frac{\tilde{\xi}(k)}{\phi}\right)\right\},
\end{aligned}
$$




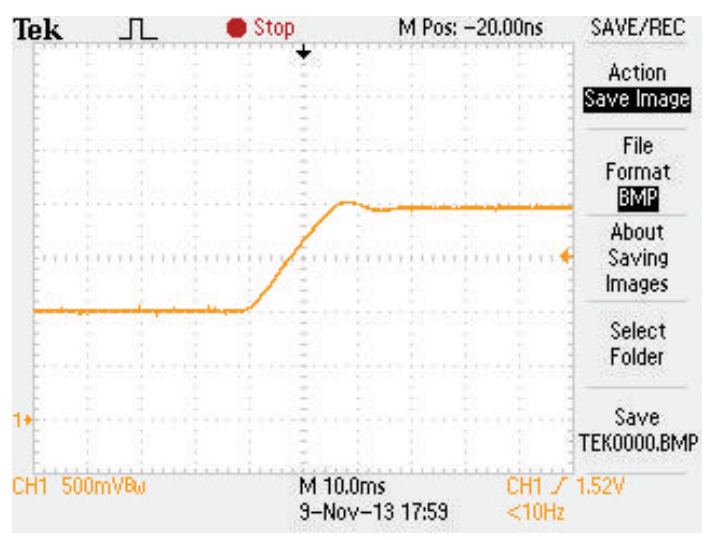

FIGURE 7: The step response of the coil current.

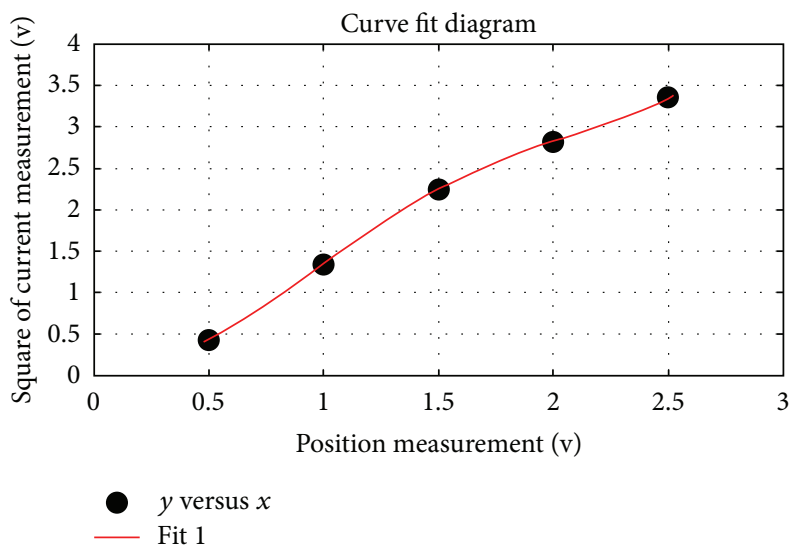

FIGURE 8: The fitting curve of the measured position and the current.

where the new parameters are $\lambda_{1}^{\prime}=\left(\lambda_{1} / g\right)(\widehat{m} / m)(1 / \Delta t), \lambda_{2}^{\prime}=$ $\left(\lambda_{2} / g\right)(\widehat{m} / m)$, and $\eta_{2}^{\prime}=\left(\eta_{2} / g\right)(\widehat{m} / m)$.

The FSMC based on (25), (26), and (14) is modified as follows:

$$
\begin{array}{r}
u_{\mathrm{fsm}}^{2}(k)=m g \hat{f}\left(v_{1}\right)\left\{1+\lambda_{1}^{\prime} \Delta v_{1}(k)+\lambda_{2}^{\prime} \varepsilon(k)\right. \\
\left.+\eta_{f}^{\prime} \cdot u_{\mathrm{fz}}\left(\frac{\widetilde{\xi}(k)}{\phi_{f}}\right)\right\},
\end{array}
$$

where the new parameter is $\eta_{f}^{\prime}=\left(\eta_{f} / g\right)(\widehat{m} / m)$.

Then the 2DFSMC (17) based on (25) and (27) is modified as follows:

$$
\begin{aligned}
u_{2 \mathrm{fsm}}^{2}(k)=m g \hat{f}\left(v_{1}\right)\{1 & +\lambda_{1}^{\prime} \Delta v_{1}(k)+\lambda_{2}^{\prime} \varepsilon(k) \\
& \left.+\eta_{2 f}^{\prime} \cdot u_{2 \mathrm{fz}}\left(\frac{\tilde{\xi}(k)}{\phi_{f_{1}}}, \frac{\Delta \tilde{\xi}(k)}{\phi_{f_{2}}}\right)\right\},
\end{aligned}
$$

where the new parameters are $\eta_{2 f}^{\prime}=\left(\eta_{2 f} / g\right)(\widehat{m} / m)$ and $\Delta \widetilde{\xi}(k)=\tilde{\xi}(k)-\tilde{\xi}(k-1)$. For the 2DFSMC method,

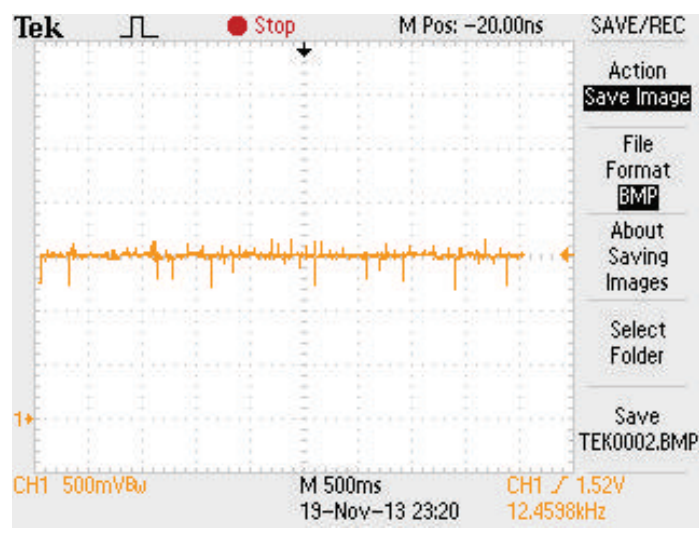

FIgURE 9: The set point response of 2DFSMC.

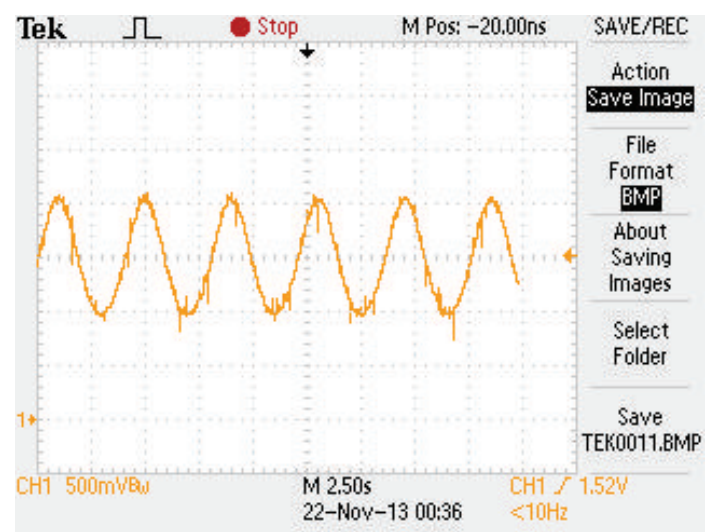

FIGURE 10: The sinusoidal response of 2DFSMC.

the experimental results are shown in Figures 9, 10, and 11. These pictures are from the digital storage oscilloscope. The vertical scale is $500 \mathrm{mV} / \mathrm{Div}$. The new control parameters are given as follows: $\lambda_{1}^{\prime}=3.08, \lambda_{2}^{\prime}=0.35, \phi_{f_{1}}=1.3, \phi_{f_{2}}=$ $1.5, \eta_{2 f}^{\prime}=0.008$. Figure 9 is the set point response of the magnet position. The set point is 1.5 Volts. From Figure 9, the magnet position is stable at equilibrium position. Because the integral error control term is included in 2DFSMC, there is no steady state error in this set point experiment. Figure 10 shows the sinusoidal response of the magnet position. This result guarantees that 2DFSMC can work in global. From Figure 10, the input signal is $1.5+0.5 \sin 0.5 \pi t$. The vertical scale is $500 \mathrm{mV} / \mathrm{Div}$ and the horizontal scale is $2.5 \mathrm{~s} / \mathrm{Div}$. The maximum output value is 2 Volts and the minimum output value is 1 Volt. The measurement output tracks the command input stably. The 2DFSMC controller is verified to stabilize the nonlinear FSMSS. From Figure 10, the sinusoidal distortion is almost none. The step response is shown in Figure 11. The command is a square wave signal. The bias signal is 1.5 Volts. The amplitude of the square wave is 0.25 Volts. From Figure 11, the system response is underdamped. This is a common phenomenon for magnetic suspension systems. For the steady state condition, there is no steady state error in this response. In the third experiment, the 2DFSMC controller is guaranteed to improve the system chattering. In experiments, 


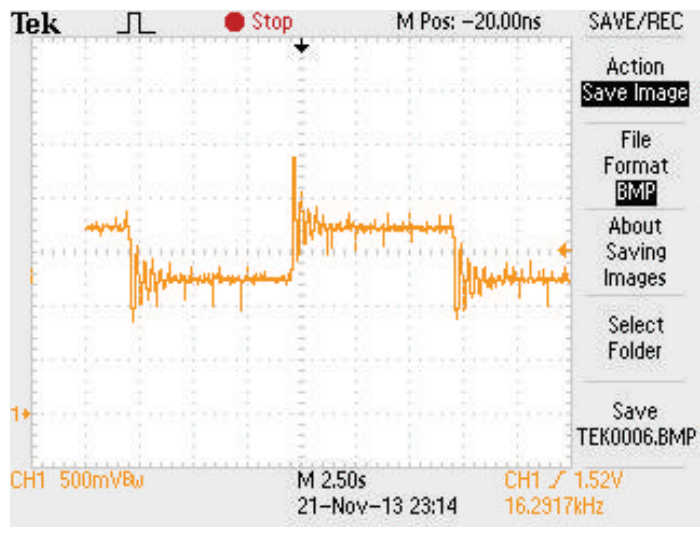

Figure 11: The step response of 2DFSMC.

the PD type control is utilized to stabilize the equilibrium positions as shown in Table 2. The stability margin is not good enough. The PD type control cannot stabilize the FSMSS globally. In nonlinear and robust control aspect, the 2DFSMC controller is superior to the $\mathrm{PD}$ type controller.

\section{Conclusion}

This paper has successfully demonstrated the 2DFSMC of a FSMSS. For 2DFSMC technique, the sliding manifold and its derivative are both considered for fuzzy rules. The more precise derivations are proposed in this paper. The Lyapunov stability proof is based on fuzzy rule based plane. The horizontal axis is represented by the sliding manifold and the vertical axis is represented by the derivative of sliding manifold. The rule table is filled out in the form of stable sliding mode control.

For the hands-on experiments, a low cost FSMSS is realized. New design circuits are proposed. The implemented driver, sensor, and control circuits are more simple, inexpensive, and effective. The control scheme is based on two-loop control. This simplifies design procedure for control of coil current. The proposed 2DFSMC improves position responses of the FSMSS effectively. This can stabilize the FSMSS globally. These experiments are appropriate for engineering education.

\section{Conflict of Interests}

The authors declare that there is no conflict of interests regarding the publication of this paper.

\section{Acknowledgments}

The authors would like to express the sincere thanks to the reviewers for their invaluable comments and suggestions. This work is supported by the National Science Council, Taiwan, Republic of China, under Grant nos. NSC 102-2221E-218-017 and NSC100-2632-E-218-001-MY3.

\section{References}

[1] J. Y. Hung, W. Gao, and J. C. Hung, "Variable structure control. A survey," IEEE Transactions on Industrial Electronics, vol. 40, no. 1, pp. 2-22, 1993.

[2] K. D. Young, V. I. Utkin, and Ü. Özgüner, "A control engineer's guide to sliding mode control," IEEE Transactions on Control Systems Technology, vol. 7, no. 3, pp. 328-342, 1999.

[3] R. Palm, "Robust control by fuzzy sliding mode," Automatica, vol. 30, no. 9, pp. 1429-1437, 1994.

[4] S.-W. Kim and J.-J. Lee, "Design of a fuzzy controller with fuzzy sliding surface," Fuzzy Sets and Systems, vol. 71, no. 3, pp. 359367, 1995.

[5] L. X. Wang, A Course in Fuzzy Systems and Control, Prentice Hall, Upper Saddle River, NJ, USA, 1997.

[6] W. Zhang, J. Li, K. Zhang, and P. Cui, "Design of magnetic flux feedback controller in hybrid suspension system," Mathematical Problems in Engineering, vol. 2013, Article ID 712764, 7 pages, 2013.

[7] W. Zhang, J. Li, K. Zhang, and P. Cui, "Decoupling suspension controller based on magnetic flux feedback," The Scientific World Journal, vol. 2013, Article ID 956790, 8 pages, 2013.

[8] K. D. Bachovchin, J. F. Hoburg, and R. F. Post, "Magnetic fields and forces in permanent magnet levitated bearings," IEEE Transaction on Magnetics, vol. 48, no. 7, pp. 2112-2120, 1999.

[9] C. E. Lin, C.-K. Yang, K.-H. Liu, and H.-L. Jou, "Improvement on drag and control performance in NCKU magnetic suspension wind tunnel," Proceedings of the National Science Council, Republic of China A: Physical Science and Engineering, vol. 24, no. 5, pp. 330-340, 2000.

[10] D. Cho, Y. Kato, and D. Spilman, "Sliding mode and classical controllers in magnetic levitation systems," IEEE Control System Magazine, vol. 13, no. 1, pp. 42-48, 1993.

[11] J.-C. Shen, " $H_{\infty}$ control and sliding mode control of magnetic levitation system," Asian Journal of Control, vol. 4, no. 3, pp. 333340, 2002.

[12] N. F. Al-Muthairi and M. Zribi, "Sliding mode control of a magnetic levitation system," Mathematical Problems in Engineering, vol. 2004, no. 2, pp. 93-107, 2004.

[13] Z.-J. Yang, K. Miyazaki, S. Kanae, and K. Wada, "Robust position control of a magnetic levitation system via dynamic surface control technique," IEEE Transactions on Industrial Electronics, vol. 51, no. 1, pp. 26-34, 2004.

[14] C.-L. Kuo, T.-H. S. Li, and N. R. Guo, "Design of a novel fuzzy sliding-mode control for magnetic ball levitation system," Journal of Intelligent and Robotic Systems: Theory and Applications, vol. 42, no. 3, pp. 295-316, 2005.

[15] H.-K. Chiang, C.-A. Chen, and M.-Y. Li, "Integral variablestructure grey control for magnetic levitation system," IEE Proceedings: Electric Power Applications, vol. 153, no. 6, pp. 809814, 2006.

[16] C.-A. Chen, H.-K. Chiang, and J.-C. Shen, "Fuzzy sliding mode control of a magnetic ball suspension system," International Journal of Fuzzy Systems, vol. 11, no. 2, pp. 97-106, 2009.

[17] F.-J. Lin, L.-T. Teng, and P.-H. Shieh, "Intelligent sliding-mode control using RBFN for magnetic levitation system," IEEE Transactions on Industrial Electronics, vol. 54, no. 3, pp. 17521762, 2007.

[18] F.-J. Lin, S.-Y. Chen, and K.-K. Shyu, "Robust dynamic slidingmode control using adaptive RENN for magnetic levitation system," IEEE Transactions on Neural Networks, vol. 20, no. 6, pp. 938-951, 2009. 
[19] J.-H. Li, "Fuzzy supervisory control of a DSP-based magnetic levitation system," Asian Journal of Control, vol. 9, no. 1, pp. 6467, 2007.

[20] J. H. Li and M. F. Wu, "Two-dimensional fuzzy sliding mode control of dsp-based magnetic levitation system," Journal of Ching-Yun University, vol. 29, no. 4, pp. 69-86, 2009.

[21] H.-J. Shieh, J.-H. Siao, and Y.-C. Liu, "A robust optimal slidingmode control approach for magnetic levitation systems," Asian Journal of Control, vol. 12, no. 4, pp. 480-487, 2010.

[22] K. H. Lundberg, K. A. Lilienkamp, and G. Marsden, "Lowcost magnetic levitation project kits," IEEE Control Systems Magazine, vol. 24, no. 5, pp. 65-69, 2004.

[23] J. H. Li, "Multiloop pid control of field-sensed magnetic suspension system," Journal of Technology, vol. 25, no. 4, pp. 315-322, 2010.

[24] J.-H. Li and J.-S. Chiou, "Robust output feedback stabiization of a field-sensed magnetic suspension system," Mathematical Problems in Engineering, vol. 2013, Article ID 192431, 7 pages, 2013.

[25] “Arminno-Cortex M3 microcontroller," http://en.innovati .com.tw/index.php.

[26] “The $\mu$ Vision4 development environment," http://www.keil .com. 


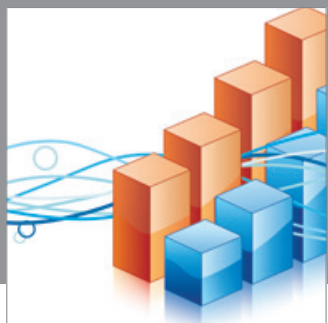

Advances in

Operations Research

mansans

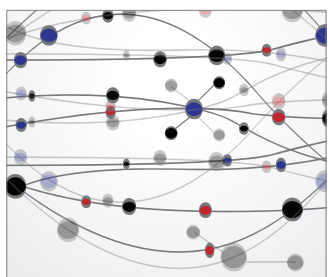

The Scientific World Journal
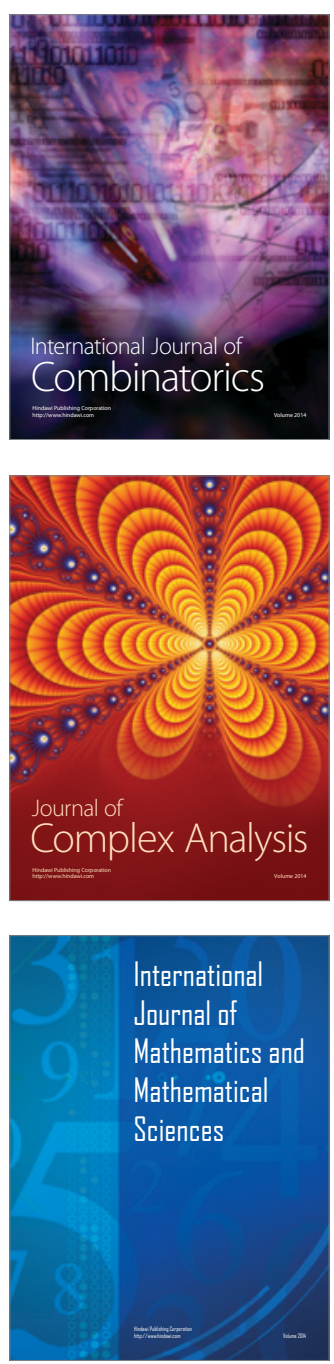
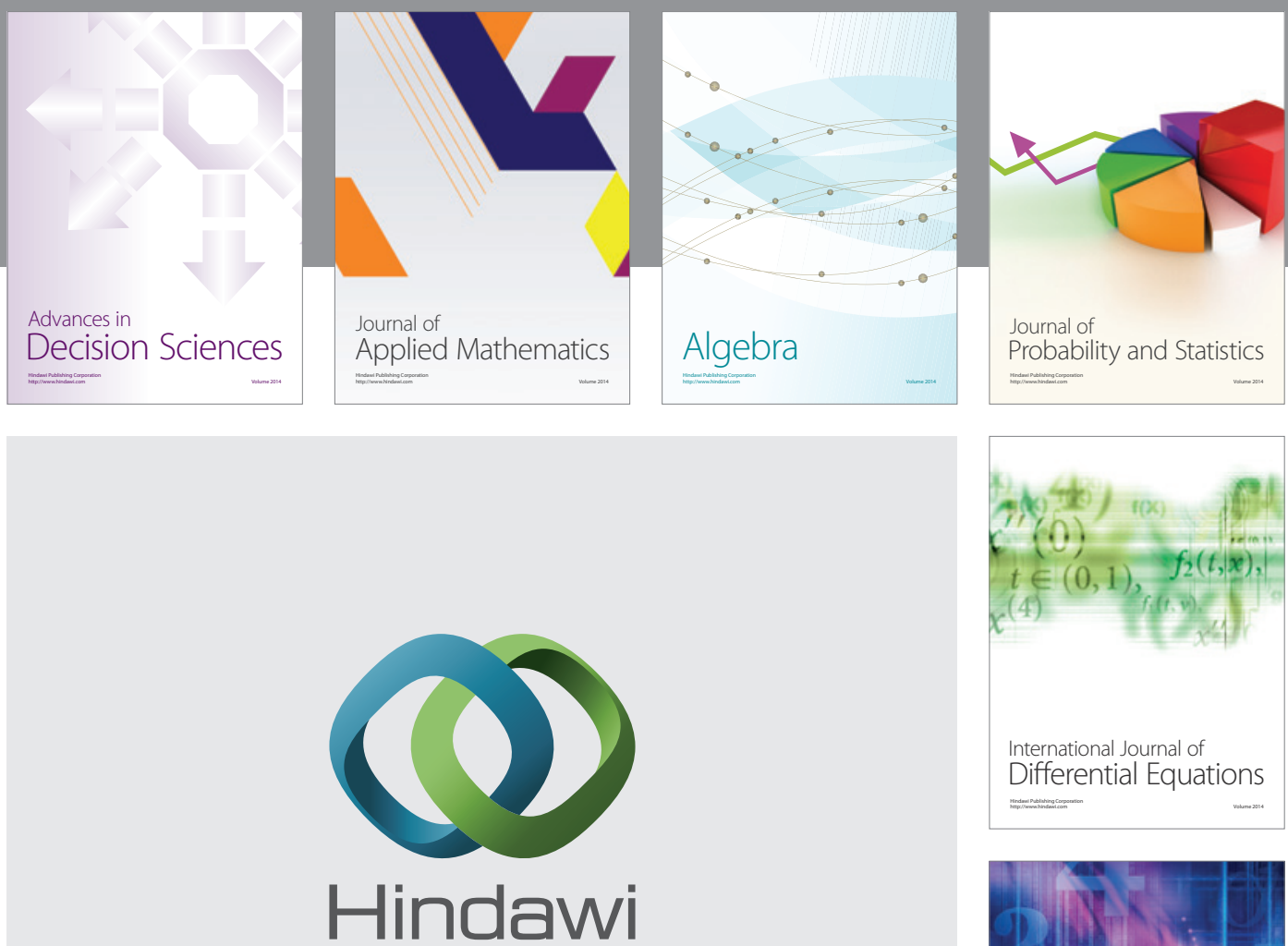

Submit your manuscripts at http://www.hindawi.com
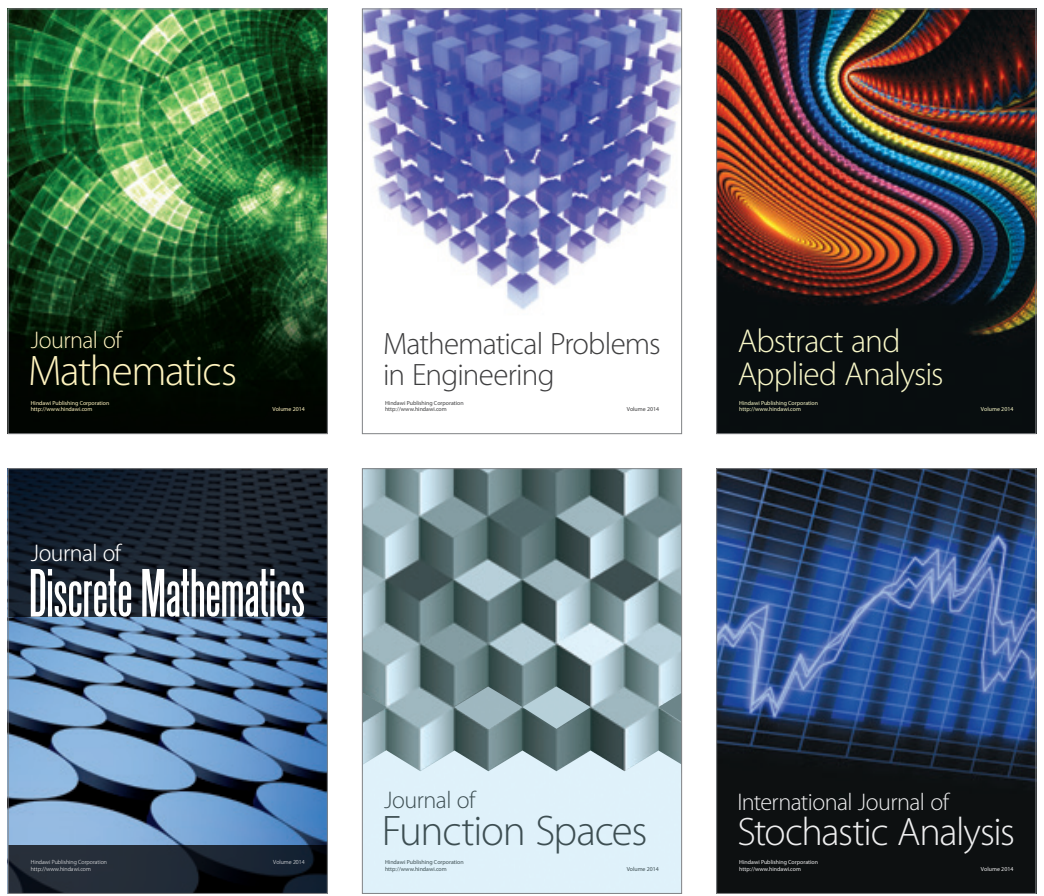

Journal of

Function Spaces

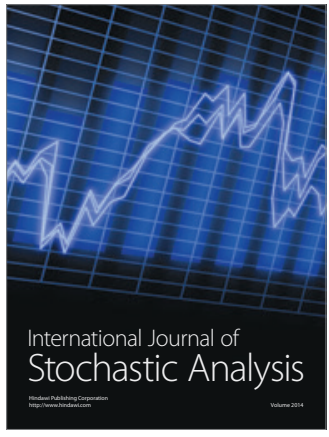

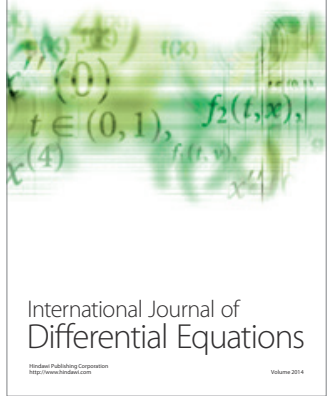
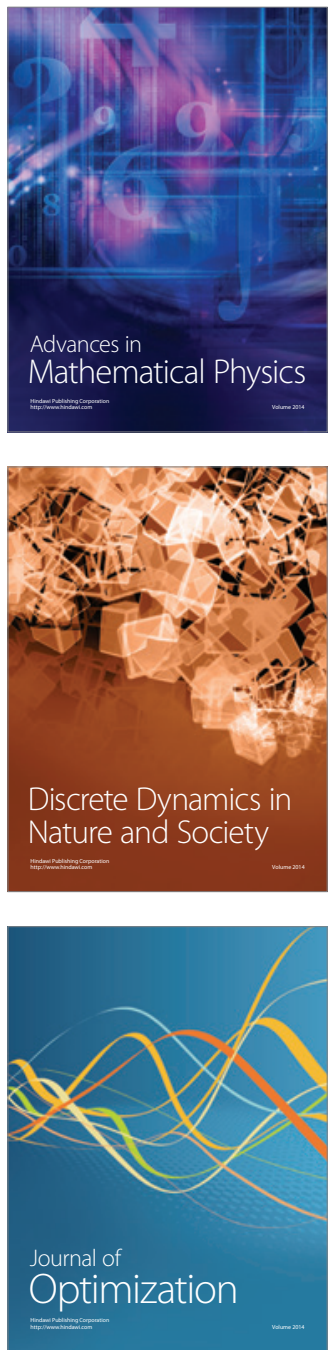